Special issue of the 2nd International Conference on Computational and Experimental Science and Engineering (ICCESEN 2015)

\title{
Laguerre Polynomial Solutions of a Class of Initial and Boundary Value Problems Arising in Science and Engineering Fields
}

\begin{abstract}
B. GÜRBÜZ* AND M. SEZER
Manisa Celal Bayar University, Department of Mathematics, Faculty of Art \& Science, Manisa, Turkey

In this study, we consider high-order nonlinear ordinary differential equations with the initial and boundary conditions. These kinds of differential equations are essential tools for modelling problems in physics, biology, neurology, engineering, ecology, economy, astrophysics, physiology and so forth. Each of the mentioned problems are described by one of the following equations with the specific physical conditions: Riccati, Duffing, EmdenFowler, Lane Emden type equations. We seek the approximate solution of these special differential equations by means of a operational matrix technique, called the Laguerre collocation method. The proposed method is based on the Laguerre series expansion and the collocation points. By using the method, the mentioned special differential equations together with conditions are transformed into a matrix form which corresponds to a system of nonlinear algebraic equations with unknown Laguerre coefficients, and thereby the problem is approximately solved in terms of Laguerre polynomials. In addition, some numerical examples are presented to demonstrate the efficiency of the proposed method and the obtained results are compared with the existing results in literature.
\end{abstract}

DOI: 10.12693/APhysPolA.130.194

PACS/topics: 02.30.Gp, 02.30.Hq, 02.70

\section{Introduction}

In this study, we are concerned with the use of Laguerre polynomials to solve some special nonlinear problems, which involve high-order nonlinear ordinary differential equations with the initial and boundary conditions. In recent years, it is well known that there exists an increasing interest in the application of the models to nonlinear problems in biology, physics and engineering. Also, the numerical solution methods of problems of these types have been developed very rapidly and intensively by many authors [1-5]. Here, the basic ideas of the mentioned studies are developed and applied to the high-order nonlinear ordinary differential equations in the form

$$
\begin{aligned}
\sum_{k=0}^{m} & \sum_{r=0}^{n} Q_{k, r}(x) y^{r}(x) \\
& +\sum_{k=1}^{m} \sum_{r=1}^{n} P_{k, r}(x) y^{(k)}(x)=f(x),
\end{aligned}
$$

under the mixed conditions

$$
\sum_{k=0}^{1}\left[a_{j k} y^{(k)}(0)+b_{j k} y^{(k)}(b)\right]=\lambda_{j}, \quad j=0,1,
$$

where $Q_{k, r}(x), P_{k, r}(x)$ and $f(x)$ are functions defined on interval $0 \leq x \leq 1 ; a, b, \lambda_{j}$ are suitable constants. The aim of this study is to get the approximate solution as the truncated Laguerre series defined by

\footnotetext{
*corresponding author; e-mail: burcu.gurbuz@cbu.edu.tr
}

$$
y(x)=\sum_{n=0}^{N} a_{n} L_{n}(x),
$$

where $L_{n}(x)$ denotes the Laguerre polynomials; $a_{n}$ $(0 \leq n \leq N)$ are unknown Laguerre polynomial coefficients, and $N$ is chosen as any positive integer such that $N \geq m$

\section{Properties of Laguerre polynomials}

A total orthonormal sequence in $L^{2}(-\infty, b]$ or $L^{2}[a,+\infty)$ can be obtained from such a sequence in $L^{2}[0,+\infty)$ by transformations $x=b-s$ and $x=s+a$, respectively. Applying the Gram-Schmidt process to the sequence defined by $\left\{\mathrm{e}^{-x / 2}, x \mathrm{e}^{-x / 2}, x^{2} \mathrm{e}^{-x / 2}, \ldots\right\}$ in $L^{2}[0,+\infty)$, we obtain an orthonormal sequence $e_{n}$. This sequence is given by

$$
e_{n}(x)=\mathrm{e}^{-x / 2} L_{n}(x), \quad n=0,1,2, \ldots
$$

where $L_{n}(x)$ are Laguerre polynomials defined by

$$
\begin{aligned}
& L_{n}(x)=\frac{\mathrm{e}^{x}}{n !} \frac{\mathrm{d}^{n}}{\mathrm{~d} x^{n}}\left(x^{n} \mathrm{e}^{-x}\right), \quad n=1,2,3 \ldots, \quad \text { or } \\
& L_{n}(x)=\sum_{r=0}^{n} \frac{(-1)^{r}}{r !}\left(\begin{array}{l}
n \\
r
\end{array}\right) x^{r}
\end{aligned}
$$

\section{Fundamental relations}

In the present study, an approximate solution in terms of linear combination of Laguerre polynomials is assumed in the form (3). For our purpose, let us consider Eq. (1) and find the matrix forms of the equation. Firstly, we can write Laguerre polynomials (4) in the matrix form 


$$
\mathbf{L}^{T}(x)=\mathbf{H} \mathbf{X}^{T}(x) \Rightarrow \mathbf{L}(x)=\mathbf{X}(x) \mathbf{H}^{T},
$$

where $\mathbf{L}(x)=\left[L_{0}(x) L_{1}(x) \ldots L_{N}(x)\right], \mathbf{X}(x)=\left[\begin{array}{lll}1 & \ldots x^{N}\end{array}\right], \mathbf{A}=\left[\begin{array}{llll}a_{0} & a_{1} & \ldots & a_{N}\end{array}\right]^{T}$ and

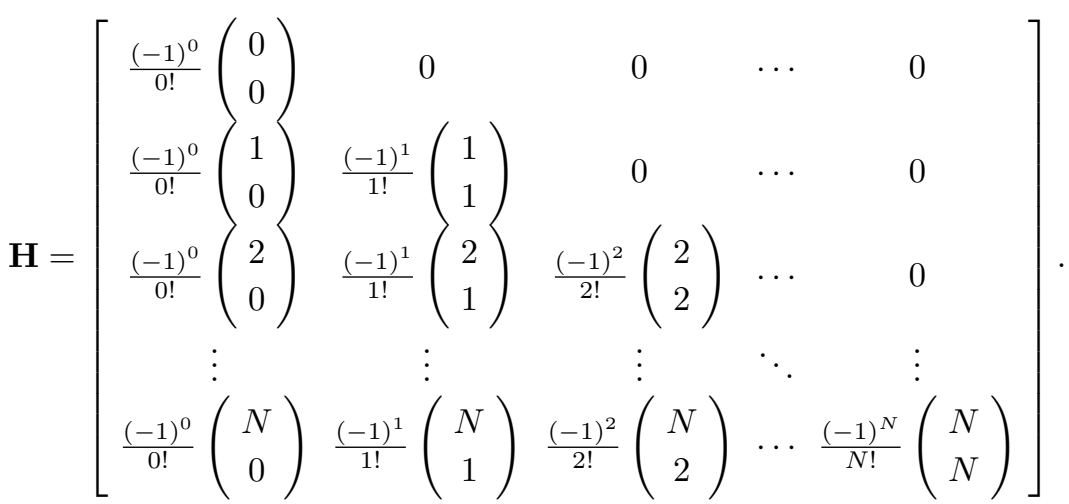

Then we set the approximate solution defined by a truncated Laguerre series (3) in the matrix form

$$
y(x)=\mathbf{L}(x) \mathbf{A} .
$$

By using the relations (5) and (6), the matrix relation is expressed as

$$
y(x)=\mathbf{X}(x) \mathbf{H}^{T} \mathbf{A} .
$$

Also, the relations between the matrix $\mathbf{X}(x)$ and its derivatives $\mathbf{X}^{\prime}(x), \mathbf{X}^{\prime \prime}(x), \mathbf{X}^{\prime \prime \prime}(x), \ldots \mathbf{X}^{(k)}(x)$ are

$$
\begin{aligned}
& \mathbf{X}^{\prime}(x)=\mathbf{X}(x) \mathbf{B}^{T}, \quad \mathbf{X}^{\prime \prime}(x)=\mathbf{X}(x)\left(\mathbf{B}^{T}\right)^{2}, \\
& \mathbf{X}^{\prime \prime \prime}(x)=\mathbf{X}(x)\left(\mathbf{B}^{T}\right)^{3}, \ldots, \mathbf{X}^{(k)}(x)=\mathbf{X}(x)\left(\mathbf{B}^{T}\right)^{k}
\end{aligned}
$$

where

$$
\mathbf{B}=\left[\begin{array}{ccccc}
0 & 0 & 0 & \ldots & 0 \\
1 & 0 & 0 & \ldots & 0 \\
0 & 2 & 0 & \ldots & 0 \\
\vdots & \vdots & \vdots & \ddots & \vdots \\
0 & 0 & 0 & N & 0
\end{array}\right]
$$

By using the relations (7) and (8), we have the matrix

relation

$$
y^{(k)}(x)=\mathbf{X}(x)\left(\mathbf{B}^{T}\right)^{k} \mathbf{H}^{T} \mathbf{A} .
$$

By substituting the Laguerre collocation points defined by

$$
x_{i}=\frac{b}{N} i, i=0,1, \ldots, N
$$

into Eq. (9), we have

$$
y^{(k)}\left(x_{i}\right)=\mathbf{X}\left(x_{i}\right)\left(\mathbf{B}^{T}\right)^{k} \mathbf{H}^{T} \mathbf{A}, \quad k=0,1, \ldots, m
$$

and the compact form of the relation (11) becomes

$$
\mathbf{Y}^{(k)}=\mathbf{X}\left(\mathbf{B}^{T}\right)^{k} \mathbf{H}^{T} \mathbf{A}, \quad k=0,1, \ldots, m
$$

where

$$
\mathbf{Y}^{(k)}=\left[\begin{array}{c}
y^{(k)}\left(x_{0}\right) \\
y^{(k)}\left(x_{1}\right) \\
\vdots \\
y^{(k)}\left(x_{N}\right)
\end{array}\right], \quad \mathbf{Y}=\left[\begin{array}{c}
y\left(x_{0}\right) \\
y\left(x_{1}\right) \\
\vdots \\
y\left(x_{N}\right)
\end{array}\right] .
$$

Similarly, by substituting the Laguerre collocation points (10) into the $y^{r}(x)$ and by using the relation (12), the matrix relation is obtained

$$
\left[\begin{array}{c}
y^{r}\left(x_{0}\right) \\
y^{r}\left(x_{1}\right) \\
\vdots \\
y^{r}\left(x_{N}\right)
\end{array}\right]=\left[\begin{array}{ccccc}
y\left(x_{0}\right) & 0 & \cdots & 0 \\
0 & y\left(x_{1}\right) & \cdots & 0 \\
\vdots & \vdots & \ddots & \vdots \\
0 & 0 & \cdots & y\left(x_{N}\right)
\end{array}\right]^{r-1}\left[\begin{array}{c}
y\left(x_{0}\right) \\
y\left(x_{1}\right) \\
\vdots \\
y\left(x_{N}\right)
\end{array}\right]=(\tilde{Y})^{r-1} \mathbf{Y}
$$

and

$$
\begin{aligned}
\tilde{\boldsymbol{Y}} & =\tilde{\boldsymbol{X}} \tilde{\boldsymbol{B}}^{T} \tilde{\boldsymbol{H}}^{T} \tilde{\boldsymbol{A}} \\
\tilde{\mathbf{X}} & =\left[\begin{array}{cccc}
\mathbf{X} & 0 & \cdots & 0 \\
0 & \mathbf{X} & \cdots & 0 \\
\vdots & \vdots & \ddots & \vdots \\
0 & 0 & \cdots & \mathbf{X}
\end{array}\right], \tilde{\mathbf{B}}^{T}=\left[\begin{array}{cccc}
\mathbf{B} & 0 & \cdots & 0 \\
0 & \mathbf{B} & \cdots & 0 \\
\vdots & \vdots & \ddots & \vdots \\
0 & 0 & \cdots & \mathbf{B}
\end{array}\right], \tilde{\mathbf{H}}^{T}=\left[\begin{array}{cccc}
\mathbf{H} & 0 & \cdots & 0 \\
0 & \mathbf{H} & \cdots & 0 \\
\vdots & \vdots & \ddots & \vdots \\
0 & 0 & \cdots & \mathbf{H}
\end{array}\right], \tilde{\mathbf{A}}=\left[\begin{array}{cccc}
\mathbf{A} & 0 & \cdots & 0 \\
0 & \mathbf{A} & \cdots & 0 \\
\vdots & \vdots & \ddots & \vdots \\
0 & 0 & \cdots & \mathbf{A}
\end{array}\right]
\end{aligned}
$$


In addition, by substituting the Laguerre collocation points (10) into the $y^{r}(x) y^{(k)}(x)$ and by using the relations (12) and (13), similarly, the compact form $(\tilde{\mathbf{Y}})^{(r)} \mathbf{Y}^{(k)}$ is obtained as [6]

$$
(\tilde{\boldsymbol{Y}})^{(r)} \boldsymbol{Y}^{(k)}=\tilde{\boldsymbol{X}} \tilde{\boldsymbol{B}}^{T} \tilde{\boldsymbol{H}}^{T} \tilde{\boldsymbol{A}} \boldsymbol{X}\left(\boldsymbol{B}^{T}\right)^{k} \boldsymbol{H}^{T} \boldsymbol{A} .
$$

\section{Method of solution}

We use the following matrix method based on the collocation points (10) to obtain Laguerre polynomial solutions of Eq. (1) under the mixed conditions (2). For this purpose, by substituting the collocation points into Eq. (1), we first have a system of equations. Then, by means of the matrix relations (11)-(14), we can convert this system to the following fundamental matrix equation

$$
\begin{aligned}
& \left\{\sum_{k=0}^{m} \sum_{r=0}^{n} \mathbf{Q}_{k, r}\left(\tilde{X} \tilde{B}^{T} \tilde{H}^{T} \tilde{A}\right)^{r} \mathbf{X}\left(\mathbf{B}^{T}\right)^{k} \mathbf{H}^{T}\right. \\
& \left.+\sum_{k=1}^{m} \sum_{r=1}^{n} \mathbf{P}_{k, r} \tilde{X} \tilde{H}^{T}\left(\tilde{B}^{T}\right)^{r} \tilde{A} \mathbf{X}\left(\mathbf{B}^{T}\right)^{k} \mathbf{H}^{T}\right\} \mathbf{A}=\mathbf{F}, \\
& \mathbf{Q}_{k, r}=\left[\begin{array}{cccc}
Q_{k, r}\left(x_{0}\right) & 0 & \cdots & 0 \\
0 & Q_{k, r}\left(x_{1}\right) & \cdots & 0 \\
\vdots & \vdots & \ddots & \vdots \\
0 & 0 & \cdots & Q_{k, r}\left(x_{N}\right)
\end{array}\right] \text {, } \\
& \mathbf{F}=\left[\begin{array}{c}
f\left(x_{0}\right) \\
f\left(x_{1}\right) \\
\vdots \\
f\left(x_{N}\right)
\end{array}\right] \\
& \mathbf{P}_{k, r}=\left[\begin{array}{cccc}
P_{k, r}\left(x_{0}\right) & 0 & \cdots & 0 \\
0 & P_{k, r}\left(x_{1}\right) & \cdots & 0 \\
\vdots & \vdots & \ddots & \vdots \\
0 & 0 & \cdots & P_{k, r}\left(x_{N}\right)
\end{array}\right] .
\end{aligned}
$$

Briefly, this equation can also be written in the form $\mathbf{W A}=\mathbf{F}$ or $[\mathbf{W} ; \mathbf{F}]$, which corresponds to a system of the $(N+1)$ nonlinear algebraic equations with the unknown Laguerre coefficients $a_{n}, n=0,1, \ldots, N$, where

$$
\begin{aligned}
& \boldsymbol{W}=\sum_{k=0}^{m} \sum_{r=0}^{n} \boldsymbol{Q}_{k, r}\left(\tilde{\boldsymbol{X}} \tilde{\boldsymbol{B}}^{T} \tilde{\boldsymbol{H}}^{T} \tilde{\boldsymbol{A}}\right)^{r} \boldsymbol{X}\left(\boldsymbol{B}^{T}\right)^{k} \boldsymbol{H}^{T} \\
& +\sum_{k=1}^{m} \sum_{r=1}^{m} \boldsymbol{P}_{k, r} \tilde{\boldsymbol{X}} \tilde{\boldsymbol{H}}^{T}\left(\tilde{\boldsymbol{B}}^{T}\right)^{r} \tilde{\boldsymbol{A}} \boldsymbol{X}\left(\boldsymbol{B}^{T}\right)^{k} \boldsymbol{H}^{T}
\end{aligned}
$$

Besides, using the relation (9) we find the fundamental matrix equation for the mixed conditions (2) as follows;

$$
\mathbf{U}_{j} \mathbf{A}=\lambda_{j} \text { or }\left[\mathbf{U}_{j} ; \lambda_{j}\right]
$$

$$
\begin{aligned}
& \mathbf{U}_{j} \mathbf{A}=\sum_{k=0}^{1}\left[a_{j k} y^{(k)}(0)+b_{j k} y^{(k)}(b)\right]\left(\mathbf{B}^{T}\right)^{k} \mathbf{H}^{T} \mathbf{A}, \\
& j=0,1, \ldots, m-1 .
\end{aligned}
$$

Consequently, replacing the $m$ rows of the augmented matrix $[\mathbf{W} ; \mathbf{F}]$ by the rows of the matrix $\left[\mathbf{U}_{j} ; \lambda_{j}\right]$, we have

$$
[\tilde{W} ; \tilde{F}], \text { or } \tilde{W} \mathbf{A}=\tilde{F}
$$

which is a system of nonlinear algebraic equations. By solving the system (17), the unknown Laguerre coefficients are computed. Thus, the approximate solution is found in the truncated Laguerre series (3) on the interval $[0, b]$.

\section{Numerical experiments}

In this section, we will consider three examples to illustrate the accuracy and efficiency of the presented method. All results have been computed using the program written in Maple 18 and graphed in Matlab 2007.

\section{Example 5.1.}

We first consider the Lane-Emden problem [7]

$$
y^{\prime \prime}(x)+\frac{2}{x} y^{\prime}(x)+y^{3}(x)=6+x^{6}, y(0)=y^{\prime \prime}(0)=0 .
$$

This problem can be written as $x y^{\prime \prime}(x)+2 y^{\prime}(x)+x y^{3}(x)=$ $6 x+x^{7}$ where $Q_{2,0}(x)=x, Q_{1,0}(x)=2, Q_{0,2}(x)=x$ and $f(x)=6 x+x^{7}$. We suppose that $y(x)$ is approximated by a truncated Laguerre series in the form $y(x)=$ $\sum_{n=0}^{2} a_{n} L_{n}(x), 0 \leq x \leq 1$. Using the procedure described in Sect. 4, the fundamental matrix relations for the equation and conditions are computed and then the Laguerre coefficients are found at the 32 th iteration as $a_{0}=\frac{3}{8}, a_{1}=\frac{1}{2}, a_{2}=\frac{1}{8}$. Therefore, the solution of this problem becomes $y(x)=x^{2}$ which is an exact solution.

\section{Example 5.2.}

We consider the Riccati equation [8]

$$
y^{\prime}(x)=y(x)-2 y^{2}(x), y(0)=1 .
$$

with the exact solution $y(x)=\frac{1}{\left(1-\mathrm{e}^{-x}\right)}$.

TABLE I

Comparison of the absolute errors for Example 5.2.

\begin{tabular}{c|c|c|c|c}
\hline \hline $\mathrm{x}$ & $\begin{array}{c}\text { Euler } \\
\text { method }\end{array}$ & $\begin{array}{c}\text { Taylor Col. } \\
\text { method } \\
\mathrm{N}=6\end{array}$ & $\begin{array}{c}\text { Suggested } \\
\text { method } \\
\mathrm{N}=6\end{array}$ & $\begin{array}{c}\text { Suggested } \\
\text { method } \\
\mathrm{N}=20\end{array}$ \\
\hline 0.1 & $1.31 \times 10^{-2}$ & $7.35 \times 10^{-5}$ & $7.05 \times 10^{-4}$ & $6.58 \times 10^{-14}$ \\
0.2 & $1.85 \times 10^{-2}$ & $7.22 \times 10^{-5}$ & $8.22 \times 10^{-5}$ & $1.70 \times 10^{-16}$ \\
0.3 & $2.04 \times 10^{-2}$ & $3.44 \times 10^{-5}$ & $3.17 \times 10^{-6}$ & $3.42 \times 10^{-16}$ \\
0.4 & $2.07 \times 10^{-1}$ & $3.22 \times 10^{-5}$ & $2.62 \times 10^{-5}$ & $2.18 \times 10^{-15}$ \\
0.5 & $2.01 \times 10^{-2}$ & $1.68 \times 10^{-5}$ & $1.20 \times 10^{-5}$ & $5.32 \times 10^{-15}$
\end{tabular}


Table I shows the solutions of the problem for different methods and various $N$. The suggested method has smaller absolute error for the same $N$ values. Similarly, in the Table I the error decreases when $N$ value is chosen large enough.

\section{Example 5.3.}

Finally, we consider the Duffing's equation of the following type $y^{\prime \prime}(x)+3 y(x)-2 y^{3}(x)=\cos (x) \sin (2 x)$ with initial conditions $y(0)=0, y^{\prime}(0)=1$ having the exact solution $y(x)=\sin (x)$.

TABLE II

Comparison of the absolute errors of Example 5.3.

\begin{tabular}{c|c|c|c|c}
\hline \hline & \multirow{2}{*}{$\begin{array}{c}\text { Exact } \\
\mathrm{x}\end{array}$} & \multicolumn{3}{|c}{ Error analysis $(|y-\widehat{y}|)$} \\
\cline { 3 - 5 } & Solution & $\mathrm{N}=5$ & $\mathrm{~N}=8$ & $\mathrm{~N}=10$ \\
\hline 0.0 & 0 & 0 & 0 & 0 \\
\hline 0.1 & 0.9983341665 & $4.62 \times 10^{-8}$ & $3.08 \times 10^{-11}$ & $4.33 \times 10^{-14}$ \\
0.2 & 0.1986693308 & $6.12 \times 10^{-7}$ & $8.72 \times 10^{-11}$ & $1.03 \times 10^{-13}$ \\
0.3 & 0.2955202067 & $4.28 \times 10^{-7}$ & $1.45 \times 10^{-11}$ & $1.66 \times 10^{-13}$ \\
0.4 & 0.3894183423 & $2.29 \times 10^{-7}$ & $1.82 \times 10^{-10}$ & $2.21 \times 10^{-13}$ \\
0.5 & 0.4794255386 & $4.23 \times 10^{-7}$ & $1.65 \times 10^{-10}$ & $2.71 \times 10^{-13}$ \\
0.6 & 0.5646424734 & $4.03 \times 10^{-7}$ & $2.62 \times 10^{-10}$ & $3.15 \times 10^{-13}$ \\
0.7 & 0.6442176872 & $3.32 \times 10^{-7}$ & $3.01 \times 10^{-12}$ & $2.29 \times 10^{-13}$ \\
0.8 & 0.7173560909 & $5.66 \times 10^{-7}$ & $3.07 \times 10^{-10}$ & $3.85 \times 10^{-13}$ \\
0.9 & 0.7833260096 & $8.87 \times 10^{-6}$ & $5.68 \times 10^{-9}$ & $9.73 \times 10^{-13}$ \\
1.0 & 0.8414709848 & $1.43 \times 10^{-5}$ & $1.22 \times 10^{-8}$ & $1.57 \times 10^{-11}$
\end{tabular}

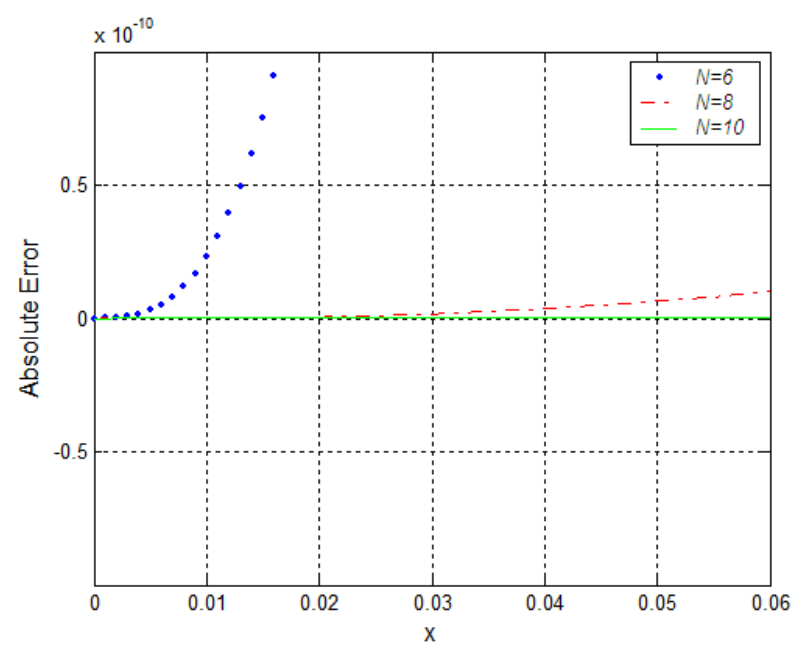

Fig. 1. Comparison of absolute errors for $N=6,8,10$.

As can be seen in Fig. 1 there are differences between errors obtained for different $N$ values. We also see that we approximate the exact solution at large $N$ values.

\section{Conclusions}

Nonlinear ordinary differential equations, such as Lane-Emden, Riccati and Duffing equations, appear frequently in modeling of physical phenomena and play an important role in the fields of science and engineering. Most of these equations have no analytical solution and so, numerical methods may be required to obtain their approximate solutions [9-13]. For this reason, the Laguerre collocation method has been presented. A considerable advantage of the method is shorter computation time and lower operation count, which results in reduction of cumulative truncation errors and in improvement of overall accuracy. For this reason, this process is much faster than the other methods. Illustrative examples with the satisfactory results are used to demonstrate the application of this method. Calculations were performed using a program written in Maple18. As a result, the power of the employed method is confirmed. The method can also be extended to another applications, but some modifications are required.

\section{Acknowledgments}

This work is founded by Manisa Celal Bayar University Department of Scientific Research Projects, with grant ref. 2014-151.

\section{References}

[1] W.T. Reid, Riccati differential equations Mathematics in science and engineering, Academic Press, New York 1972, p. 86.

[2] Ş. Yüzbaşı, Int. J. Comp. Math. 89, 2180 (2012).

[3] F. Karakoç, H. Bereketoğlu, Int. J. Comput. Math. 86, 914 (2009).

[4] A. Arıkoğlu, I. Özkol, Appl. Math. Comput. 174, 1216 (2006).

[5] B. Gürbüz, M. Gülsu, M. Sezer, Math. Comput. Appl. 16, 267 (2011).

[6] A. Akyüz-Daşcıoğlu, H. Çerdik-Yaslan, Appl. Math. Comput. 217, 5658 (2011).

[7] S. Yüzbaş, M. Sezer, Math. Comput. Model. 57, 1298 (2013).

[8] M. Gülsu, M. Sezer, J. Appl. Math. 27, 821 (1996).

[9] A. El-Safty, M.S. Salim, M.A. El-Khatib, Int. J. Comput. Math. 80, 509 (2003).

[10] F. Karakoç, H. Bereketoğlu, Int. J. Comput. Math. 86, 914 (2009).

[11] E. Yusufoğlu, Appl. Math.Comput. 177, 572 (2006).

[12] M.M. Hosseini, Appl. Math. Comput. 175, 1685 (2006).

[13] J.C. Jiao, Y. Yamamoto, C. Dang, Y. Hao, Comput. Math. Appl. 43, 783 (2002). 